Jahre nach Inkrafttreten des BSHG, die Hilfe zur Pflege 28\% des Gesamtaufwandes der Sozialhilfe und fast die Hälfte des Aufwandes für die Hilfe in besonderen Lebenslagen. ${ }^{308}$ Für die Haus- bzw. Familienpflege hingegen wurden auch Ende der 1990er Jahre der Mangel verbindlicher Rechtsgrundlagen und eine unzureichende Finanzierung auf seiten der freien Verbände beklagt, obwohl der Bedarf dafür unverändert hoch veranschlagt wurde. ${ }^{309}$

\title{
5. Hilfen für Behinderte
}

Mit der „Eingliederungshilfe für Behinderte“ wurde in die Fürsorgereform ein sozialpolitisches Feld einbezogen, das, lange vernachlässigt, besonders viel innovatives Potential besaß. Neben rein medizinischen Maßnahmen und Unterhaltssowie Pflegeleistungen sollte Hilfe zur schulischen und beruflichen Ausbildung bis hin zur Suche nach einem Arbeitsplatz und darüber hinaus zur sozialen Integration gewährt werden, um dem Behinderten ein möglichst selbständiges Leben zu ermöglichen.

Traditionell hatten Eingliederungshilfen für (Körper-)Behinderte oder „Krüppel“, wie es zeitgenössisch hieß, im deutschen Sozialleistungssystem ihren Platz vor allem in der gewerblichen Unfallversicherung und der Kriegsopferfürsorge mit dem Grundsatz, statt dauerhafter Rentengewährung möglichst die Wiedereingliederung in den Arbeitsmarkt zu erreichen. ${ }^{310}$ Neben medizinischen Hilfen zur Verhütung von Invalidität, wie sie auch die Rentenversicherung von Anfang an kannte, gehörten dazu die Versorgung mit orthopädischen Hilfsmitteln, Ausbildungsmaßnahmen und Hilfe bei der Arbeitsvermittlung. ${ }^{311}$ Diesem Ziel diente auch das Reichsgesetz über die Beschäftigung Schwerbeschädigter von 1923, das für schwerbeschädigte „Kriegskrüppel“ und gleichgestellte Unfallopfer Einstellungsquoten vorschrieb und Kündigungsschutz gewährte. Soziale Hilfe für nicht kriegs- oder arbeitsbedingt Körperbehinderte hingegen hatte lange vor allem die freie Wohlfahrtspflege geleistet.

1920 erklärte das preußische Krüppelfürsorgegesetz (andere Länder folgten) die Fürsorge für hilfsbedürftige „Krüppel“ zur Pflichtaufgabe der öffentlichen Fürsorge und führte im Interesse der Früherkennung eine Meldepflicht von minderjährigen Körperbehinderten ein; das Gesetz übertrug die stationäre Fürsorge den

gerade wenn man ihr Verhältnis zum gesamten Sicherungssystem ins Auge faßt - ihre wohl umfangreichste Aufgabe vorfindet“(S.215), und schätzte die Zahl allein der künftigen Dauerpflegefälle grob auf 0,5 bis 1,1 Mio. (S.217f.); vgl. Schellhorn, Sozialhilfe, S.243f.; Giese, 25 Jahre, S.376. Die Einführung der Pflegeversicherung 1995 hat die Sozialhilfeträger erheblich entlastet, die Hilfe zur Pflege aber nicht überflüssig gemacht, da die Versicherungsleistungen die tatsächlichen Kosten der Pflege keineswegs immer decken; vgl. Oestreicher/Schelter/Kunz/Decker, Bundessozialhilfegesetz, S. 32a.

308 Vgl. Wirtschaft und Statistik 1965, S. 605, Tabelle 2.

309 Vgl. Angelika Maier, Familienhilfe; Falk-Lutz-Bachmann, Familienpflege.

310 Vgl. NDV 41 (1961), S.190f.; Sachße/Tennstedt, Geschichte, Bd.2, S. 90ff., 132; Kolb, Rehabilitationsrecht, S. 1389.

311 Vgl. dazu die Vorschriften über soziale Fürsorge für Kriegsopfer in den RGr. (\$S 24ff.). 
leistungsfähigeren Landesarmenverbänden (später Landesfürsorgeverbänden), die ambulante den Städten und Kreisen, so daß bald vielerorts eigene „Krüppelfürsorgestellen“ tätig wurden. ${ }^{312}$ 1924/25 regelten RFV und RGr. auch das Leistungsrecht und schrieben im Rahmen des notwendigen Lebensbedarfs neben der medizinischen Rehabilitation für hilfsbedürftige „Krüppel“, Blinde und Taubstumme auch „Hilfe zur Erwerbsbefähigung“ als Pflichtleistung vor. ${ }^{313}$ Denn, so die amtlichen Erläuterungen von 1931, es sei „die wirksamste, würdigste und im Endergebnisse sparsamste Hilfe, die Kräfte der Schwererwerbsbeschränkten dem Wirtschaftsleben nutzbar zu machen“. 314 Durchgeführt wurde die Fürsorge häufig in „Krüppelheimen“ der freien Wohlfahrtspflege oder in arbeitsfürsorgerischen Einrichtungen und geschützten Werkstätten in städtischer Trägerschaft. Da aber die Ausbildung sich meist auf „behindertentypische“ handwerkliche Berufe wie Bürstenbinder, Korbmacher oder Schneider beschränkte, gelang die Eingliederung in den ersten Arbeitsmarkt vielfach nicht.

Während des Nationalsozialismus wurde die „Krüppelfürsorge“ organisatorisch in den Aufgabenbereich der staatlichen Gesundheitsämter einbezogen und die Zahl der Fürsorgestellen weiter ausgebaut. Doch die Fürsorge für „Krüppel“ paßte kaum zur „Herrenmenschen“-Ideologie; statt dessen wurde die Anzeigepflicht nun zum Instrument von Zwangssterilisation und „Euthanasie“.315

Nach dem Ende des Zweiten Weltkriegs erforderte schon die bloße Zahl der Behinderten eine Forcierung der Hilfsmaßnahmen: Bei der Volkszählung 1950 gaben rund 1,66 Mio. Personen im Bundesgebiet körperliche oder geistige Gebrechen an; ein methodisch nur bedingt möglicher Vergleich mit dem Stand von 1925 ergab, daß bei fast gleichem Abstand zum jeweiligen Kriegsende sich die absolute Zahl der Behinderten mehr als verdoppelt hatte. ${ }^{316}$ Die Zahl der Körperbehinderten betrug rund 1,02 Mio.; anders als 1925 (21\%) hatte die weitaus meisten dieser Menschen (69,3\%) der Krieg zu Behinderten gemacht - statistischer Ausdruck der menschlichen Konsequenzen der modernen Kriegführung, die sich auch im relativ hohen Anteil von kriegsbedingt schwerbehinderten Zivilistinnen (11\%) an allen schwerbehinderten Frauen widerspiegelte.

In der sozialpolitischen Gesetzgebung für Behinderte dominierte daher zunächst die Hilfe für Kriegsopfer, die zivile Bombengeschädigte mitumfaßte, wobei man mit dem Bundesversorgungsgesetz und dem Schwerbeschädigtengesetz von 1953 an Weimarer Vorbilder anknüpfte, aber die Zivilbehinderten weitgehend ausschloß. ${ }^{317}$ Für die übrigen zivilen Behinderten - mit Ausnahme der Unfallver-

312 Preußisches Gesetz betr. die öffentliche Krüppelfürsorge vom 6.5.1920 (Preußische Gesetzsammlung, S. 280); dazu NDV 34 (1954), S.339f.

313 \ 6 Nr.b, e RGr. Die Durchführung der Schwerbeschädigtenfürsorge wie der sozialen Fürsorge für Kriegsopfer oblag ebenfalls den Fürsorgeverbänden, vgl. $\ 1$ RFV.

314 Abgedruckt bei Muthesius, Grundlagen, S. 84.

315 Vgl. Sachße/Tennstedt, Geschichte, Bd.3, S.173ff.; zu den Bestimmungen des GVG siehe NDV 34 (1954), S. 340.

316 Vgl. Hudemann, Sozialpolitik, S.526ff.; NDV 34 (1954), S. 342ff.; auch Jochheim u.a., Rehabilitation, S. 567ff.

317 Vgl. Hudemann, Sozialpolitik, S. 537f., sowie 450ff., zur Entwicklung in den einzelnen Besatzungszonen. Das Schwerbeschädigtengesetz bezog neben den Opfern von Krieg 
sicherten - hingegen fehlten solche Sozialleistungen ganz oder waren so uneinheitlich geregelt, daß sie nur ungenügend zum Tragen kamen ${ }^{318}$ : „Wie in einem Vergrößerungsglas", resümierte der damalige Behindertenfunktionär und spätere SPD-Bundestagsabgeordnete Eugen Glombig, „zeigten sich auf dem Gebiet der Rehabilitation alle Mängel [des sozialen Sicherungssystems]: Kompetenzwirrwarr, Ungleichbehandlung der Behinderten je nach der Ursache der Behinderung, Mangel an geeigneten Rehabilitationseinrichtungen, völlige Vernachlässigung der von Kindheit und Jugend an Behinderten". ${ }^{319}$ Im Sommer 1955 schätzte die Bundesregierung die Zahl allein der nicht versorgten Körperbehinderten (ohne Blinde, Taubstumme) auf rund $200000, \mathrm{zu}$ denen neben seinerzeit nur notdürftig behandelten Flüchtlingen immer mehr Opfer von Verkehrsunfällen und (nach mehreren Epidemien) von Kinderlähmung gehörten. ${ }^{320}$

Allgemeine Not und knappe öffentliche Mittel, allmählich auch der Mangel an Arbeitskräften führten dazu, daß Hilfe zur (Wieder-)Eingliederung lange vor allem als Hilfe zur Erwerbsbefähigung diskutiert wurde, seltener auch als Hilfe zu größerer Selbständigkeit im Alltagsleben. ${ }^{321}$ Die Diskussionen konzentrierten sich dabei auf die Körperbehinderten, während geistig/psychisch Behinderte mangels eigener Lobby und sicher auch wegen der Nachwirkungen nationalsozialistischer Propaganda nur am Rande oder aber bis Ende der fünfziger Jahre primär unter dem Gesichtspunkt der „Bewahrung“ ins Blickfeld gerieten. ${ }^{322}$

Nachdem bereits im Frühling 1950 verschiedene Körperbehinderten-Organisationen und die westdeutschen Hauptfürsorgestellen den Entwurf für ein Bundesfürsorgegesetz für Körperbehinderte vorgelegt hatten und zusammen mit den kommunalen Spitzenverbänden im Dezember beim Bundesinnenministerium vorstellig geworden waren, wurde diese Initiative von der SPD aufgegriffen und

und NS-Verfolgung sowie nach Arbeitsunfällen Behinderten nur die „Zivilblinden“ mit ein. Zu deren Sonderstellung s.u.; ferner Rudloff, Schatten, S. $364 \mathrm{ff}$.

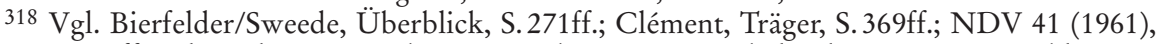
S.191ff. Schon die Frage, ob eine angeborene Körperbehinderung eine Krankheit im Sinne der RVO sei, führte zu langwierigen Streitigkeiten zwischen BFV und Krankenkassen, so daß die Betroffenen oft keine rechtzeitige Hilfe erhielten; vgl. Anlage 1a zum Niederschriftsentwurf der Sitzung des Studienkreises „Soziale Neuordnung“ am 3./4.12. 1954, ADW, HGSt, SP-S XXIIIc I/O. Darüber hinaus waren auch für Krankenversicherte die Leistungen auf 26 Wochen begrenzt.

319 Glombig, Rehabilitation, S. 214f.; ferner NDV 34 (1954), S.341; Hasenclever, Jugendhilfe, S. 189.

320 Vgl. die Begründung zum Entwurf des Körperbehindertengesetzes vom 11.7.1955, S.7f., BT, 2. Wp. 1953, Anlagen, Bd.36, Drs. 1594.

321 Vgl. das DV-Gutachten über eine Teilreform des Fürsorgerechts in: Rundschreiben 1946, S. 51; NDV 28 (1948), S. 43, 64; so auch das Verständnis in der Kabinettsvorlage des BMA vom 7.4.1955, in: Kabinettsprotokolle. Ministerausschuß für die Sozialreform, S. 212f. Zur Ausweitung der Bedeutung von „Integration“ im Laufe der fünfziger Jahre vgl. Rudloff, Schatten, S. 350ff., 404.

322 Vgl. etwa Petersen, Unterbringung; ferner die Beiträge in der Arbeitsgruppe V des Fürsorgetages 1952, NDV 32 (1952), S. 424ff. Diese Privilegierung der Körperbehinderten wurde etwa vom Reichsbund auch forciert, vgl. Glombig, Regelung, S. 83. Zur sozialpolitischen Randstellung der geistig Behinderten vgl. Rudloff, Schatten, S. 404ff. 
Anfang April 1951 von allen Fraktionen des Bundestags die Vorlage eines Gesetzes beantragt. ${ }^{323}$ Bis dahin sollten allerdings noch vier Jahre vergehen.

$\mathrm{Zu}$ dieser Verzögerung trug neben der Vielzahl der beteiligten Bundesressorts und den widersprüchlichen Interessen der betroffenen Verbände und Körperschaften die Tatsache bei, daß die Berechtigung und Notwendigkeit eines Bundesgesetzes von einzelnen Ländern überhaupt bestritten wurde, zumal auch hier wieder die Frage der Stellung der Gesundheitsämter eine wichtige Rolle spielte. ${ }^{324}$ Darüber hinaus leisteten viele Landesfürsorgeverbände als Hauptträger der vorgesehenen Leistungen und die Landkreise erheblichen Widerstand. ${ }^{325}$ Selbst nachdem der Entwurf schon in den Bundestag eingebracht worden war, versuchte der DLT, noch auf höchster Ebene die Rückstellung der Entwürfe für das KBG wie für das THG bis zur allgemeinen Fürsorgereform zu betreiben. ${ }^{326}$ Der Entwurf sah nämlich großzügige Leistungen und Einkommensgrenzen vor, außerdem besondere Beratungsstellen, die als Eingriff in die eigene Verwaltungshoheit betrachtet wurden, und drohte somit ein gefährliches Präzedens für die Fürsorgereform zu werden. Doch im Innenministerium war man sich bewußt, daß man den Bundestag nicht ewig hinhalten und angesichts neuer Früherkennungsmethoden nicht weiter wertvolle Zeit im Interesse der Betroffenen verstreichen lassen konnte. ${ }^{327}$

Außerdem vernetzten sich die Überlegungen für eine Neuordnung der Körperbehindertenfürsorge bald mit den Diskussionen um die „Sozialreform“, in denen die Eingliederung der Behinderten - oder wie es in neuer Terminologie bald hieß: die „Rehabilitation“ - ein wichtiges Thema bildete ${ }^{328}$ : als Ausweis für einen modernen Sozialstaat, der auch die Belange seiner verschiedenartig behinderten Bürger ernst nahm und ihnen ein selbstbestimmtes Dasein statt bloßer Heimverwahrung zugestand ${ }^{329}$, ebenso wie als Instrument der Hilfe zur Selbsthilfe und damit

323 Vgl. Antrag der SPD-Fraktion vom 2. 2. 1951, BT, 1. Wp. 1949, Anlage, Bd. 9, Drs. 1869; Sitzung des Bundestages am 4.4.1951, ebenda, Sten. Ber., Bd.6, S. 4944f.; zur Vorgeschichte Jellinghaus, Betrachtungen und Forderungen, S. 4ff., $20 \mathrm{ff}$.

324 Vgl. Jellinghaus, Betrachtungen und Forderungen, S. 5ff.; Bundesratssitzung am 24.6. 1955, S. 176, BR 1955, Sten. Ber.

325 Vgl. die Anlagen 1 und 1a zum Niederschriftsentwurf der Sitzung des Studienkreises „Soziale Neuordnung“ am 3./4.12.1954, ADW, HGSt, SP-S XXIIIc I/O.

326 Vgl. DLT-Präsident Seebich an den Bundesinnenminister am 30.8.1954; ders. an Globke am 6. 9.1955; ferner DLT an die Mitglieder des Bundesratsausschusses für Innere Angelegenheiten am 13.6.1955; Niederschrift über die Sitzung des DLT-Sozialausschusses am 25./26.7.1955, BAK, B 172/444-01/1.

327 Vgl. Anlage 1 zum Niederschriftsentwurf der Sitzung des Studienkreises „Soziale Neuordnung" am 3./4.12.1954, ADW, HGSt, SP-S XXIIIc I/90.

$328 \mathrm{Vgl}$. Bierfelder/Sweede, Überblick; Fürsorge und Sozialreform, S.343ff.; NDV 36 (1956), S. 43ff.; Marx, Die Städte zur Neuordnung, S. 46ff.; Neuordnung des Fürsorgerechts, S. 197ff.; Frandsen, Eingliederungshilfe, S. $21 \mathrm{f}$.

329 In den „Grundlagen eines Sozialplanes der SPD“ von 1952 spielte die „Säule“ der Berufssicherung dabei die entscheidende Rolle, vgl. Richter, Sozialreform, Bd.6 G II, S.1, 3. Zur zentralen Bedeutung, die man im BMI der Rehabilitation im künftigen Sozialsystem beimaß, siehe Kitz, Gegenwartsfragen, S. 331, 333. 
als Gegensteuerung zur „Rentensucht“. 330 Dabei wurde immer wieder ein großer Nachholbedarf gegenüber den USA und vor allem Großbritannien konstatiert, wo umfangreiche gesetzliche Rehabilitationsprogramme die berufliche Eingliederung von Behinderten - bei zumindest in Großbritannien vergleichbarer Größenordnung - ungleich wirksamer förderten. ${ }^{331}$ Auch die Internationale Arbeitskonferenz hatte sich seit Kriegsende verstärkt dieses Problems angenommen und detaillierte Empfehlungen vor allem zur beruflichen Rehabilitation unterbreitet und dabei die rückhaltlose Abkehr vom Kausalitätsprinzip gefordert. ${ }^{332}$

Statt der vielen uneinheitlichen Regelungen zur Rehabilitation wurde in den "Sozialreform“-Debatten daher deren systematische rechtliche und institutionelle Neuordnung gefordert und auch im Bundesinnenministerium favorisiert. ${ }^{333}$ Ziel war dabei, die Prävention deutlich zu verstärken, möglichst alle Behinderten einzubeziehen und die Hilfe statt bisher kausal (nach der Ursache der Behinderung) nun final (nach der jeweils notwendigen Hilfeform) zu organisieren. Wie so viele in der Sache wenig kontroverse „Sozialreform“-Überlegungen scheiterten auch diese an der Frage der institutionellen Umsetzung. Achinger schlug eine neue Sonderbehörde zum Schutz der bedrohten Arbeitskraft vor; im Gegensatz dazu favorisierten Auerbach und später der SPD-Sozialplan von 1957, die Rothenfelser Denkschrift, der DGB und die Ortskrankenkassen unterschiedliche kooperative Modelle, in denen die verschiedenen Aufgaben der (medizinischen, beruflichen) Rehabilitation jeweils bei einem Träger zusammengefaßt werden sollten. Das Bundesarbeitsministerium hingegen wollte unter Berufung auf den Beirat nur die Zusammenarbeit der verschiedenen Träger innerhalb des bestehenden Systems, das seine Rehabilitationsmaßnahmen allerdings insgesamt verstärken und dafür höhere Staatszuschüsse erhalten müsse. ${ }^{334} \mathrm{Ganz}$ anders die Fürsorgefunktionäre der kommunalen Spitzenverbände: Dem sozialreformerischen Doppelziel einer Erweiterung kommunaler Kompetenzen wie eines Ausbaus des Bedarfsprinzips

330 Vgl. Achinger auf dem Fürsorgetag 1952, NDV 32 (1952), S. 469ff.; so dann auch einhellig, wenn auch unterschiedlich motiviert, in den verschiedenen Kabinettsvorlagen des BMA wie von dessen Gegenspielern (BMF, BMI, BMW) zur Sozialreform vom Frühling 1955, Soziale Sicherheit 4 (1955), S. 279ff.; ferner Kabinettsprotokolle, Ministerausschuß für die Sozialreform, S.212f.; die Entschließung des CDU-Bundesparteitags vom April 1956, Richter, Sozialreform, Bd. 6 G I, S. 17.

331 Vgl. NDV 34 (1954), S. 168ff.; Bierfelder/Sweede, Überblick, S. $273 f f$.

332 Vgl. Empfehlung Nr.99 der Internationalen Arbeitsorganisation betr. die berufliche Eingliederung und Wiedereingliederung der Behinderten vom 22.6.1955, BABl. 1955, S.669, der die Bundesregierung Ende Dezember 1956 zustimmte, vgl. NDV 37 (1957), S. 63, 67; ferner NDV 38 (1958), S. 191ff.

333 Vgl. Scheffler vor der Sozialministerkonferenz am 29.5.1956, Kurzfassung des Referats, BAK, B 106/20652.

334 Vgl. Glombig, Rehabilitation, S. 214ff.; mit den entsprechenden Literaturangaben Bierfelder/Sweede, Überblick, S.273ff.; Achinger u.a., Neuordnung der sozialen Leistungen, S.69ff.; ferner die Kabinettsvorlage des BMA vom 7.4.1955, in: Kabinettsprotokolle, Ministerausschuß für die Sozialreform, S. 210ff.; Hockerts, Entscheidungen, S. 272f.; die Beirats-Empfehlungen vom Juni 1955, BABl. 1955, S. 540; anders jedoch der Fürsorgeausschuß des Beirats am 15./16.6.1956, der ein umfassendes Rehabilitationsgesetz forderte; Niederschrift, ADW, HGSt 6769; Sozialplan, S. 80, 127. 
entsprechend forderte Bangert vom DLT ähnlich wie Hildegard Schräder vom DST Anfang 1955, die „Zusammenfassung aller Vorbeugungs- und Rehabilitationsmaßnahmen bei der öffentlichen Fürsorge“, was „zu einem wesentlich wirksameren und sparsameren Einsatz der heute auf viele Bereiche sozialer Leistungen verzettelten öffentlichen Mittel führen“ könnte ${ }^{335}$; an den Kosten dieser Maßnahmen sollten sich dann die anderen Sozialleistungsträger beteiligen - damit lag Bangert ganz auf der Linie des Bundesfinanzministeriums. ${ }^{336}$

Eine derartige Neuregelung der Rehabilitation wäre allerdings weder mit den anderen Sozialleistungsträgern noch dem Arbeitsminister zu machen gewesen. Doch auch die verschiedenen Kooperationsmodelle stießen auf Bedenken der Sozialversicherungsträger, die um ihre Unabhängigkeit fürchteten. ${ }^{337}$ So blieben die Forderungen nach einem eigenen Rehabilitationsgesetz Makulatur, und erst 1974 wurden die Leistungen der verschiedenen Träger (mit Ausnahme der Sozialhilfe!) gesetzlich einander angeglichen und die privilegierte arbeitsrechtliche Stellung auf alle Schwerbehinderten ausgedehnt. ${ }^{338}$

Statt dessen gab es nur partielle Verbesserungen für die jeweilige Klientel innerhalb des bestehenden Leistungssystems ${ }^{339}$ : Mit der Rentenreform von 1957 wurde neben der erweiterten medizinischen Rehabilitation erstmals auch die Berufsförderung als „Regelleistung“ eingeführt, sofern mit deren Erfolg zu rechnen war; einen Rechtsanspruch auf Rehabilitation - wie von der SPD gefordert - gab es auch weiterhin nicht. ${ }^{340} 1956 / 57$ wurde die Bundesanstalt für Arbeitsvermittlung erstmals, wenn auch unpräzise, zur beruflichen Rehabilitation von geistig und körperlich Behinderten verpflichtet, allerdings ohne ihnen einen Rechtsanspruch einzuräumen. ${ }^{341}$ Auch im Fürsorgerecht waren die Rehabilitationsleistungen etwas weiter ausgebaut worden, indem das FÄG von 1953 u.a. die Pflicht zu erzieherischen Hilfen auch für behinderte Minderjährige verschärfte und den körperlich Behinderten bessere Ausbildungsmöglichkeiten eröffnete. ${ }^{342}$ Das Problem der vielen Körperbehinderten und ihrer Familien, deren Einkommen über den fürsorgerischen Grenzen lag, die aber durch teure Heilverfahren und Hilfsmittel oder Anstaltskosten finanziell überfordert waren, blieb ungelöst. Ebenso unbefriedigend blieben die Maßnahmen zur Früherkennung von Behinderungen, vor allem in den nicht ehemals preußischen Gebieten ohne Meldepflicht.

335 Niederschrift über die Sitzung des DLT-Sozialausschusses am 4.2.1955, BAK, B 172/444-01/1; vgl. ferner Schräder, Städte.

336 Vgl. Vermerk Bangert vom 24.1.1955, BAK, B 172/444-01/1; ferner die Kabinettsvorlage des BMF vom 13.5. 1955, in: Soziale Sicherheit 4 (1955), S. 281, 284.

337 Vgl. NDV 36 (1956), S. 43ff.

338 Vgl. Kolb, Rehabilitationsrecht, S. 1389f.; Glombig, Rehabilitation, S. 224ff.

339 Vgl. NDV 37 (1957), S. 63ff.; Glombig, Rehabilitation, S. 218ff.; Hockerts, Entscheidungen, S. $356 f$.

340 Vgl. SS 1235-1244 RVO in der Fassung vom 23.2.1957, BGBl. I S. 45; dazu kritisch Collmer, Bedeutung, S. 251f.

341 Vgl. $\int 39$ Abs. 3 AVAVG in der Fassung vom 3.4.1957, BGBl. I S. 322; NDV 39 (1959), S. $25 f$.; 41 (1961), S. 186ff., $193 f$.

342 Vgl. Art. IV, VII FÄG, BGBl. I S. 967, bzw. die Neufassung der $\ 6$, d) und e) sowie \11d RGr. 
Parallel zu diesen Diskussionen und immer wieder durch Anfragen der SPD im Bundestag angetrieben ${ }^{343}$, hatte die Sozialabteilung an Entwürfen für das geforderte Gesetz gearbeitet, diese aber bis Anfang 1955 noch nicht bis zur Kabinettsreife gebracht. Im März 1955 durch ein „Ultimatum“ der SPD-Bundestagsfraktion unter Druck gesetzt ${ }^{344}$, legte das Innenministerium schließlich am 11.Juli 1955 den ganze 19 Paragraphen umfassenden Entwurf für ein Gesetz zur Körperbehindertenfürsorge dem Bundestag vor. ${ }^{345}$ Dessen Ausschüsse für Fürsorge und Gesundheitswesen berieten den Entwurf daraufhin fast anderthalb Jahre, bis dieser Anfang Dezember 1956 vom Bundestag in zweiter und dritter Lesung verändert, auf Veranlassung des Bundesrates noch dem Vermittlungsausschuß vorgelegt und schließlich (kurz nach der Rentenreform) am 8. Februar 1957 vom Bundestag verabschiedet wurde. ${ }^{346}$ Am 1. April des Jahres trat das Körperbehindertengesetz (KBG) in Kraft. ${ }^{347}$

Auf der Basis einer dem medizinischen Erkenntnisstand entsprechenden Definition der „Körperbehinderung“ baute das KBG bundesweit die Pflichtaufgaben der öffentlichen Fürsorge aus und vereinheitlichte deren organisatorischen Aufbau und Zuständigkeiten sowie die Meldepflicht. Danach war es bereits Pflicht der Fürsorge, „drohende Körperbehinderung durch rechtzeitiges Einschreiten zu verhüten“, vorhandene Behinderung zu beseitigen, insbesondere die berufliche Eingliederung der Behinderten vorzubereiten bzw. deren „Leiden durch Gewährung angemessener Bildung und Pflege zu erleichtern“ $(\mathbb{\$} 2)$. Damit folgte auch die Bundesregierung der Auffassung, daß „die Förderung der Körperbehinderten [...] nicht ausschließlich unter dem Gesichtspunkt ihres Einsatzes als notwendige Arbeitskräfte betrachtet werden“ dürfe, sondern daß „im Rahmen einer ganzheitlichen Betrachtungsweise des Menschen [...] auch der Körperbehinderte zu seinem eigenen Wohl Gelegenheit erhalten sollte, durch Entwicklung seiner Gesamtpersönlichkeit, einschließlich der ihm verbliebenen Fähigkeiten ein vollwertiges Glied der Gesellschaft zu werden“.348

343 Vgl. die Antwort des BMI vom 18.4.1952 auf eine Kleine Anfrage der SPD-Fraktion, BT, 1. Wp. 1949, Anlage, Bd.17, Drs. 3314; Kleine Anfrage der SPD vom 6. 4.1954, BT, 2. Wp. 1953, Anlagen, Bd.28, Drs. 438, und Antwort des BMI vom 23.4.1954, ebenda, Bd.29, Drs. 479; Mündliche Anfrage Abg. Bennemann vom 21.1.1955, ebenda, Bd.33, Drs. 1157, sowie die Antwort Innenminister Schröders im Bundestag am 27.1.1955, S. 3313, ebenda, Sten. Ber., Bd. 23.

344 Einen Antrag der SPD vom 9.3.1955, den Entwurf bis zum 1.7.1955 vorzulegen, BT, 2. Wp. 1953, Anlagen, Bd.34, Drs. 1246, hatte der Bundestag am 27.4.1955 an den Fürsorgeausschuß verwiesen, ebenda, Sten. Ber., Bd.24, S. 4306. Mitte Mai war der Gesetzentwurf daraufhin endlich vom Bundeskabinett verabschiedet worden, vgl. Niggemeyer am 8.7.1955; ebenda, Bd.26, S. 5455.

345 Vgl. BT, 2. Wp. 1953, Anlagen, Bd.36, Drs. 1594; Sitzung des Bundestages am 13.7. 1955, ebenda, Sten. Ber., Bd. 26, S. 5465.

346 Vgl. BT, 2. Wp. 1953, Sten. Ber., Bd. 35, S. $10929 f$.

347 Gesetz über die Fürsorge für Körperbehinderte und von einer Körperbehinderung bedrohte Personen (KBG) vom 27.2.1957, BGBl. I S.147.

348 Begründung zum Entwurf des KBG vom 11.7.1955, S. 9, BT, 2.Wp. 1953, Anlagen, Bd.36, Drs. 1594. 
Diese Leistungen sollten jetzt auch Behinderten mit angeborenen Leiden zugute kommen, nicht hingegen Blinden, Taubstummen oder anderweitig versorgten Unfall- und Kriegsbeschädigten; da die jetzige oder künftige ,dauernde wesentliche Beeinträchtigung der Erwerbsfähigkeit‘ entscheidendes Kriterium war, betraf das Gesetz auch nicht alte Menschen oder nur vorübergehend Erwerbsunfähige. ${ }^{349}$ Die Leistungen umfaßten Heilverfahren, orthopädische Versorgung, ,angemessene" Schulausbildung sowie Fort- oder Umschulung und richteten sich im übrigen nach dem allgemeinen Fürsorgerecht. Von entscheidender Bedeutung waren die neuen Kostenregelungen: Die Rückzahlungspflichten des Hilfeempfängers wurden weitgehend aufgehoben, vor allem aber die Einkommensgrenzen großzügig geregelt. Der Behinderte hatte nur dann zu den Kosten von Heilverfahren und größeren Hilfsmitteln einen Beitrag zu leisten, wenn seine steuerpflichtigen Einkünfte die Krankenversicherungspflichtgrenze für Angestellte (1957: 500 DM) überstiegen. ${ }^{350}$ Dadurch wurde die Einkommensgrenze nicht nur praktisch dynamisiert, sondern auf ein weit über späteren Sozialhilfestandards liegendes, schon versorgungsähnliches Niveau gehoben und die bislang problematische Gruppe der „Minderbemittelten“ einbezogen. ${ }^{351}$ Ursprünglich war diese Einkommensgrenze nicht für ambulante Heilungskosten vorgesehen, im Bundestag aber mit knapper Mehrheit auf Antrag der SPD auch auf diese Maßnahmen ausgedehnt worden. ${ }^{352}$ Anderthalb Jahre später allerdings wurde mit dem THG diese Grenze gegen den Widerstand der SPD-Fraktion auf dem aktuellen Stand von 660 DM eingefroren. 353

Da die Hilfe bislang oft an der Leistungsschwäche kleiner Kreise und Gemeinden gescheitert war, machte das KBG die Landesfürsorgeverbände zu den Hauptträgern der Fürsorge für Körperbehinderte und verpflichtete diese notfalls zur Vorleistung, um notwendige Maßnahmen nicht durch Streitigkeiten mit Krankenkassen zu verzögern. Die Bundesländer hatten mindestens einen Landeskörperbehindertenarzt zu bestellen und die Gesundheitsämter weiterhin die ärztliche Beratung durchzuführen. Im Interesse eines systematischen Rehabilitationsverfahrens mußten diese einen umfassenden Heil- und Eingliederungsplan erstellen. Für alle Länder wurde eine Meldepflicht von minderjährigen Körperbehinderten eingeführt. Diese Regelung war die mit Abstand umstrittenste des parlamentarischen Verfahrens gewesen und hatte, so Willy Könen (SPD) rückblickend, „z.T. groteske

349 Inhaltlich knüpfte das Gesetz also an den traditionellen, mittlerweile aber pejorativ gebrauchten Begriff des „Krüppels“ an, bezog allerdings „Seelentaube“ (sensorische Aphasie) und "Hörstumme“ (motorische Aphasie) mit ein (vgl. $\$ 1$ KBG). Der - von der SPD sogar lieber explizit fixierte - Ausschluß alter Menschen ist im Zusammenhang mit den Forderungen nach einer stärkeren Verankerung der Rehabilitation in der Rentenversicherung zu sehen, vgl. den Abg. Bärsch (SPD) vor dem Bundestag am 6.12. 1956, BT, 2. Wp. 1953, Sten. Ber., Bd. 33, S. 9832 f.

350 Diese Grenze erhöhte sich für jeden unterhaltenen Angehörigen um 10\%, maximal jedoch $50 \%$, vgl. $\$ 10 \mathrm{KBG}$.

351 Vgl. Glombig, Rehabilitation, S. 221.

352 Vgl. Bundestagssitzung am 6.12. 1956, BT, 2. Wp. 1953, Sten. Ber., Bd. 33, S. 9850.

353 Vgl. $\ 32$ THG, BGBl. I S. 523. 
Auseinandersetzungen“ veranlaßt. ${ }^{354}$ Ursprünglich hatte die Bundesregierung wie die Körperbehindertenverbände auch eine Meldepflicht von Erwachsenen einführen und den Kreis der meldepflichtigen Personen erheblich erweitern wollen, um dadurch auch bisher nicht behandelte Körperbehinderte einzubeziehen. ${ }^{355}$ Diese Regelung stieß auf heftigen Widerstand der Ärzte, da eine Meldung an das Gesundheitsamt ohne Einverständnis des Behinderten gegen die ärztliche Schweigepflicht verstoße. ${ }^{356}$ Nach langwierigen Verhandlungen mit dem überwiegend von Ärzten beschickten Gesundheitsausschuß hatte der Fürsorgeausschuß des Bundestages bereits die Bestimmungen etwas entschärft ${ }^{357}$; doch im Bundestag erreichten schließlich Mitglieder des Gesundheitsausschusses aus der Regierungskoalition, daß die Meldepflicht für Erwachsene wieder gestrichen wurde und auch auf Minderjährige und Unmündige nur anzuwenden war bei dem Verdacht, daß deren Sorgeberechtigte ihre Pflichten vernachlässigten. 358 Während das Gesetz im Bundestag schließlich fast einstimmig beschlossen wurde, fand es bei den Behindertenverbänden nur begrenzt Beifall, da es nicht die von ihnen gewünschte stärkere Annäherung an die Versorgungsleistungen für Kriegsbeschädigte gebracht hatte. ${ }^{359}$

Noch während der parlamentarischen Beratungsphase des KBG hatte Gottschick für das künftige Bundesfürsorgegesetz eine besondere „Hilfe für Behinderte" vorgesehen. ${ }^{360}$ Deren Leistungen stimmten weitgehend mit denen des KBGEntwurfs überein, sollte das KBG doch ebenfalls in das allgemeine Fürsorgegesetz eingearbeitet werden. Die damit verbundenen Ziele wurden bereits erörtert: Beseitigung der mangelnden Systematik der Rehabilitation innerhalb des Fürsorgerechts und Verhinderung künftiger Sondergesetze, boten diese doch ein gefürchtetes Einfallstor für das seit Jahren von verschiedenen Behindertengruppen geforderte einkommensunabhängige Pflegegeld. ${ }^{361}$

Erklärter Gegner dieses Einbaus war der Reichsbund, denn damit wäre die Chance, das KBG allmählich zu einem Versorgungsgesetz zu entwickeln, ein für

354 Könen, Weg, S. 405.

355 Vgl. $\ 3$ des KBG-Entwurfs vom 11.7.1955, BT, 2. Wp. 1953, Anlagen, Bd.36, Drs. 1594.

356 Vgl. BldW 104 (1957), S. 3; zu den teilweise polemischen Äußerungen der Ärzteverbände siehe Könen und Niggemeyer vor dem Bundestag am 6.12.1956, BT, 2. Wp. 1953, Sten. Ber., Bd. 33, S. $9837 \mathrm{ff}$.

357 Vgl. den Schriftlichen Bericht des Bundestags-Fürsorgeausschusses vom 19.11.1956, S. 2ff., BT, 2. Wp. 1953, Anlagen, Bd. 46, Drs. 2885.

358 Argumentiert wurde im Bundestag dabei von seiten der Mitglieder des Gesundheitsausschusses wie von der FDP, die eine völlige Abschaffung der Meldepflicht verlangte, vor allem mit dem verfassungsmäßigen Recht auf körperliche Unversehrtheit, das einen Behandlungszwang verhindere und somit die Meldepflicht obsolet mache; auch die SPD hatte schließlich aufgrund ähnlicher Bedenken die Beschränkung auf Minderjährige beantragt; vgl. die Bundestagssitzung am 6.12.1956, BT, 2. Wp. 1953, Sten. Ber., Bd.33, S. 9832 ff.; nebst Umdrucken Nr. 856f., 863, 873, ebenda, S. $8964 f f$.

${ }^{359} \mathrm{Vgl}$. Glombig, Kritik.

360 Vgl. [Konzept] „C. Sozialhilfe“ vom 29.11.1955, BAK, B 106/20648.

361 Vgl. Vermerk Bangert vom 4.2.1957, BAK, B 172/444-01/4; Neuordnung des Fürsorgerechts, S. $207 f$. 
alle Mal verbaut gewesen. ${ }^{362}$ Überdies fürchtete man hier Leistungsverschlechterungen für die eigene Klientel, nicht weniger aber die Nähe zu den Fürsorgeempfängern; schließlich sei man bislang in vielen Ländern nicht durch die Fürsorgeämter, sondern durch die Fürsorgestellen für Schwerbeschädigte betreut worden, was sich „für die Betroffenen außerordentlich segensreich ausgewirkt“ habe. ${ }^{363}$ Außerdem plante die Sozialabteilung von Anfang an, sämtliche Behindertengruppen einzubeziehen, neben den Blinden, Gehör- und Sprachgeschädigten, wie vom Reichsbund befürwortet, auch geistig und psychisch Behinderte, zumal diese, so Scheffler, „in gleicher oder ähnlicher Weise schutzbedürftig“ seien. ${ }^{364}$ Dies ging dem Reichsbund zu weit, könne doch „die gemeinsame Behandlung körperlich und geistig Behinderter in der Öffentlichkeit mißverstanden werden“ und würde „auf jeden Fall von den Körperbehinderten als Abwertung aufgefaßt“.365

Problematischer für die Gesetzesmacher der Sozialabteilung war allerdings auch hier der anhaltende Widerstand der Kollegen der Gesundheitsabteilung und führender Medizinalbeamter der Länder, die den Einbau allgemeiner gesundheitsrechtlicher Bestimmungen, die ebenso für Behinderte außerhalb der Fürsorge galten (Meldepflicht, Landesarzt usw.), strikt ablehnten. ${ }^{366}$ Doch auch hier konnte sich die Sozialabteilung mit ihrem Ziel der Rechtszusammenfassung durchsetzen und wählte aus „optischen Gründen“367 wie bei der Tuberkulosehilfe den Weg, die gesundheitsrechtlichen Vorschriften bis zur Verabschiedung eines neuen Gesundheitsgesetzes in einem Sonderabschnitt des BSHG-Entwurfs zu verankern. ${ }^{368}$

Der Referentenentwurf vom Juli 1958 faßte den Begriff der Behinderten weitestmöglich und definierte die Hilfen großzügig und noch klarer als im KBG mit einem Rechtsanspruch versehen. ${ }^{369}$ Anders als in den anderen Sozialgesetzen be-

362 Vgl. Glombig, Rehabilitation, S. 222.

363 Stellungnahme des Reichsbundes [5.11.1958], BAK, B 106/9686; vgl. ferner dessen Stellungnahme zum BSHG-Regierungsentwurf o.D., PA, Gesetzesmaterialien III/349 A 3. Etwas weniger scharf, aber in der Sache ähnlich der andere große Behindertenverband, der VdK, vgl. dessen Stellungnahme zum Regierungsentwurf vom 14.10.1960, ebenda; anders die Fachorganisation „Deutsche Vereinigung der Körperbehindertenfürsorge“ in ihrer Stellungnahme vom 17.11.1958, BAK, B 106/9686.

364 Gerhard Scheffler, Neuordnung, S.36. Zum umfassenden Ansatz des BMI vgl. [Konzept] „C. Sozialhilfe" vom 29.11.1955 und den BSHG-Teilentwurf vom 25.5.1956, \$S 63ff., BAK, B 106/20648; Vermerk Referat V A 4 vom 22.6.1956, BAK, B 106/9689; dazu auch die Niederschriften über die Sitzungen des Arbeitsausschusses für Fragen der Fürsorge am 3./4.5.1957, ADW, HGSt 6769, und der Gruppe IV des DV-Studienkreises „Soziale Neuordnung“ am 16.7.1957, ADW, HGSt, SP-S XXIIIc I/O.

365 Stellungnahme des Reichsbundes [5.11.1958], BAK, B 106/9686.

366 Vgl. Abteilungsleitervorlage vom 25.6.1957, BAK, B 106/29652; Vermerke Referat V A $4 \mathrm{zu}$ Stellungnahmen der Abteilung IV (Gesundheitsabteilung) vom 18.2., 5.3. und 28.5.1958; Abteilung IV an Abteilung V, 11.6.1958, BAK, B 106/29643; Vermerk Karl zum Entwurf eines Sozialhilfegesetzes [27.4.1959], BAK, B 106/20644.

367 Vermerk Referat V A vom 12.6.1957, BAK, B 106/9789/2.

368 Vgl. Unterabschnitt 1 des Abschnitts 15 des BSHG-Entwurfs 7/1958, BAK, B $106 / 20643$.

369 Vgl. $\iint 55 f f .$, ebenda. 
zog die Sozialabteilung auch die soziale Rehabilitation ein und zeigte sich damit auf der Höhe des aktuellen Diskussionsstandes. ${ }^{370}$ Dem modernen Verständnis von „Rehabilitation“ als koordiniertem und kontinuierlichem Prozeß, bei dem die verschiedensten Stellen zusammenwirken müßten, trug der Entwurf mit einem ausführlichen Maßnahmenkatalog Rechnung, der insofern noch über das KBG hinausging, als u.a. Hilfe bei der Arbeitssuche, nachgehende Hilfe und höhere Leistungen zum Lebensunterhalt vorgesehen waren. Konnte der Behinderte keine Tätigkeit ausüben, sollte er von Pflege weitgehend unabhängig gemacht und sollten ihm möglichst ,angemessene Bildung und Anregungen kultureller oder sonstiger Art vermittelt werden“. ${ }^{371}$ Mit der Streichung des Kriteriums der „dauernden wesentlichen Beeinträchtigung der Erwerbsfähigkeit“ wurde klargestellt, daß die Sozialhilfe auch für die Rehabilitation alter Menschen, etwa nach einem Verkehrsunfall, zuständig war. ${ }^{372}$

Daneben enthielt der Entwurf jedoch eine bedeutsame Verschlechterung für die Körperbehinderten: Er fixierte die Einkommensgrenze für stationäre Maßnahmen, ambulante Heilbehandlung und Beschaffung teurer Hilfsmittel bei 600 DM und koppelte sie damit wieder von der Grenze der Krankenversicherungspflicht ab. Da statt der steuerpflichtigen Einkünfte nun das Netto-Einkommen zugrundezulegen war, bedeutete dies vorerst u.U. sogar eine Verbesserung; doch mittelfristig wurde so die (automatische!) Anbindung an die Entwicklung des allgemeinen Lebensstandards wieder verabschiedet und damit ein entscheidender Fortschritt des KBG zurückgenommen. ${ }^{373}$

Die allgemeinen gesundheitsrechtlichen Vorschriften im Sonderabschnitt 15 des Entwurfs entsprachen weitgehend denjenigen des KBG und bezogen sich ausdrücklich nur auf körperlich Behinderte. Eine Meldepflicht auch für geistig und psychisch Behinderte hielt man - wohl ebenso aus historischen Gründen wie angesichts der jüngsten parlamentarischen Erfahrungen - für „eine Überspannung “. ${ }^{374}$ Das von den Behinderten-Vertretern immer wieder geforderte feste, möglichst einkommensunabhängige Pflegegeld für Schwerbehinderte 375 enthielt auch der BSHG-Entwurf nicht. Dabei hatte der Bundestag bereits Anfang 1957 auf Antrag von CDU/CSU und FVP die Bundesregierung aufgefordert, die Möglichkeit eines Pflegegeldes auch für andere als blinde Pflegebedürftige zu prüfen. ${ }^{376}$ Doch Anfang 1961 vertrat Staatssekretär Anders vom Innenministerium den Standpunkt, daß dem Bund zumindest für ein versorgungsrechtliches Pflege-

370 Vgl. Beiträge und Leitsätze der Untergruppe „Rehabilitation“, in: Neuordnung des Fürsorgerechts, S. $197 \mathrm{ff}$.

371 \55 Abs. 2 des BSHG-Entwurfs 7/1958, BAK, B 106/20643.

372 Vgl. Frandsen, Eingliederungshilfe, S. 23.

373 Vgl. \80 BSHG-Entwurf 7/1958, BAK, B 106/20643.

374 Gerhard Scheffler, Neuordnung, S. 36.

${ }^{375} \mathrm{Vgl}$. die Stellungnahmen des VdK vom 15.10.1958 und der Deutschen Vereinigung zur Förderung der Körperbehindertenfürsorge vom 17.11.1958, BAK, B 106/9686, sowie des Reichsbunds [o.D.] zum Regierungsentwurf, PA, Gesetzesmaterialien III/349 A 3 .

376 Vgl. Bundestagssitzung am 21.1. 1957 sowie Umdruck 912, BT, 2. Wp. 1953, Sten. Ber., Bd. 35, S. $10599,10617$. 
geld die Gesetzgebungsbefugnis fehle und wenn überhaupt, nur eine einkommensabhängige Lösung im Rahmen des Fürsorgerechts möglich sei. ${ }^{377}$

Wie schon bei anderen Hilfearten in der Überarbeitungsphase bis zum zweiten Referentenentwurf zu beobachten, ließ sich auch die großzügige Ausgestaltung der Eingliederungshilfe trotz positiver Resonanz bei vielen Behindertenorganisationen, Wohlfahrtsverbänden und im DV nicht halten. ${ }^{378}$ Vor allem der generelle Rechtsanspruch für die verschiedensten Behinderten stieß auf erheblichen Widerstand, nicht nur bei den auch hier erwartungsgemäß opponierenden Landkreisen und Landgemeinden, sondern auch bei den Vertretern der Länder und bezeichnenderweise bei der Zentralen Spruchstelle für Fürsorgestreitsachen: Ein Rechtsanspruch auf Rehabilitation für geistig und psychisch Behinderte gehe zu weit, er müsse vielmehr auf klar definierte Behindertengruppen entsprechend der bisherigen Rechtslage und Fürsorgepraxis begrenzt sein, damit auch die Zuständigkeit der überörtlichen Träger klar geregelt und der Anlaß für künftige Klagen minimiert werden. ${ }^{379}$ Hier spielten auch Erfahrungen mit dem KBG eine wichtige Rolle: Die Rentenversicherungsträger, Krankenkassen, aber auch Lastenausgleichs- und manche Versorgungsämter hatten nämlich unter Berufung auf die Verpflichtung der Landesfürsorgeverbände zur Rehabilitation eigene Kann-Leistungen verweigert. ${ }^{380}$ Eine höchstrichterliche Klärung war bislang nicht erfolgt. Eine Ausweitung des fürsorgeberechtigten Personenkreises barg daher in den Augen sparsamer Fürsorgevertreter zu viele Gefahren, die auch durch die klare Formulierung des Nachrangs der Sozialhilfe in $\mathbb{S} 3$ des Entwurfs nicht gebannt würde.

In Zusammenarbeit mit einigen Vertretern der Landesfürsorgeverbände und der Spruchstelle, unter ihnen auch Heinz Keese, legte Gottschick mit dem Referentenentwurf vom März 1959 dann eine Regelung vor, die fast wie bisher einen Rechtsanspruch nur noch „Körperbehinderten, von einer Körperbehinderung bedrohten Personen, Blinden, hochgradig Sehschwachen, Hörgeschädigten, Sprachgeschädigten und Personen, deren geistige Kräfte schwach entwickelt sind“, einräumte. ${ }^{381}$ Für andere „Personen mit einer körperlichen, geistigen oder seelischen

377 Vgl. Antwort des BMI vom 2.2.1961 auf eine Kleine SPD-Anfrage, BT, 3. Wp. 1957, Anlagen, Bd.73, Drs. 2460.

378 Vgl. die Stellungnahmen des VdK vom 15.10.1958, des DV [vom 5.11.1958], der Inneren Mission/Hilfswerk vom 14.11.1958, der Deutschen Vereinigung für Körperbehindertenfürsorge vom 17.11.1958, der Gesellschaft zur Förderung der Hör- und Sprachgeschädigten vom 24.11.1958, BAK, B 106/9686; kritischer allerdings die AWO am 14.10.1958, ebenda; ohne größere Einwände zu den $\mathbb{S} 55 \mathrm{ff}$. auch der DST am 27.11.1958 und der DPW am 22.12.1958, ebenda.

379 Vgl. Niederschriften über die Besprechungen mit Vertretern der obersten Landessozialbehörden am 21./22.10. und 6.11.1958, LAS Abt. 761 Nr. 8874; die Stellungnahmen der bayerischen kommunalen Spitzenverbände vom 14.10.1958, des DLT vom 17.11.1958, des DGT vom 1.12.1958, BAK, B 106/9686; Kurzprotokoll über die Sitzung der Zentralen Spruchstelle für Fürsorgestreitsachen am 12.12.1958, BAK, B 106/20644.

380 Vgl. entsprechende Berichte auf einer Besprechung der leitenden Fürsorgereferenten der Länder am 12./13.6.1958, Niederschrift, BAK, B 106/20132; zu den Einzelheiten: Schaudienst, Subsidiarität; NDV 41 (1961), S. $195 f$.

381 \36 des BSHG-Entwurfs 3/1959, BAK, B 106/20646. 
Behinderung" war die Hilfe nur noch als Kann-Leistung vorgesehen. Dabei, so ein Mitarbeiter der Inneren Mission, müßte eigentlich gerade den Menschen, die aus anderen als körperlichen Gründen in ihrer beruflichen Leistungsfähigkeit eingeschränkt und bisher am seltensten von den Versicherungs- und Versorgungsträgern erfaßt waren, von der Fürsorge geholfen werden. ${ }^{382}$ Für diese Behinderten kam hinzu, daß sie jetzt auch nicht mehr von der besonderen Einkommensgrenze profitieren konnten: Eltern eines nach einer Hirnhautentzündung geistig behinderten Kindes etwa hatten also deutlich mehr an eigenem Einkommen einzusetzen, ehe ihr Kind Eingliederungshilfen erhielt, als Eltern eines Kindes mit angeborener Trisomie 21 („Mongolismus“). ${ }^{383}$ Für nicht unter die Definition des KBG fallende Körperbehinderte etwa mit einem rheumatisch bedingten schweren Herzfehler galt das gleiche.

Die besondere Einkommensgrenze selbst wurde, wie dargelegt, deutlich herabgesetzt: auf $500 \mathrm{DM}$, um sie der aktuellen Grenze des KBG anzugleichen. ${ }^{384} \mathrm{Da}$ auch die allgemeine Einkommensgrenze auf den anderthalbfachen Eckregelsatz vermindert wurde, war jetzt für ambulante Eingliederungshilfen als erhöhte allgemeine Grenze der zweifache Regelsatz (plus 80 DM Familienzuschlag) vorgesehen. ${ }^{385}$ Auch die Aufgabenstellung der Eingliederungshilfe wurde auf Drängen der Landesfürsorgeverbände wieder beschränkt und blieb dabei sogar noch hinter derjenigen des KBG zurück: Statt umfassender sozialer Integration sollte sie „vor allem“ dazu dienen, „dem Behinderten die Ausübung eines Berufs oder einer sonstigen angemessenen Tätigkeit zu ermöglichen oder ihn wenigstens unabhängig von Pflege zu machen“. ${ }^{386}$ Derart wieder reduziert wurden diese Bestimmungen fast unverändert in den Regierungsentwurf und schließlich auch vom Gesetzgeber weitgehend übernommen. ${ }^{387}$

Laut Berechnung des Bundesinnenministeriums würde die Eingliederungshilfe aber auch so zu den Hilfen mit der größten Kostensteigerung gehören: Vor allem, weil außer den klassischen Körperbehinderten nun auch weitere Behindertengruppen unter die besondere Einkommensgrenze fallen würden, ging man hier von einer Verdoppelung der bisherigen jährlichen Kosten von 18 Mio. DM aus. ${ }^{388}$ So hatte die Einschränkung des Personenkreises auch im parlamentarischen Verfahren Bestand, obwohl der Bundesrat dafür plädierte, auch durch Krankheit oder

382 Vgl. Vermerk Heun vom 5. 5.1959, ADW, HGSt, SP-S XXV 1: 160-1/1.

383 Vgl. Gottschick, Bundessozialhilfegesetz, 1962, zur Abgrenzung des Personenkreises nach $₫ 37$ Abs. 1 und 2, S. $166 f f$.

384 Der Familienzuschlag wurde allerdings auf 80 DM erhöht; vgl. $\ 58$ des BSHG-Entwurfs 3/1959, BAK, B 106/20646.

$385 \mathrm{Vgl}$. $\ 57$ (ebenda) nur für Maßnahmen der Schul- und Berufsbildung, sowie dann allgemein für alle ambulanten Hilfen mit Ausnahme der Heilbehandlung $\mathbb{} 76$ des BSHGRegierungsentwurfs vom Februar 1960, BT, 3. Wp. 1957, Anlagen, Bd. 67, Drs. 1799.

386 \$36 Abs. 3 BSHG-Entwurf 3/1959, BAK, B 106/20646; vgl. Stellungnahme der AG der LFV, BAK, B 106/20644.

387 Vgl. SS 37ff., 116ff. des BSHG-Regierungsentwurfs 1960, BT, 3. Wp. 1957, Anlagen, Bd. 67, Drs. 1799; $\int \mathbb{S} 39 \mathrm{ff} ., 123 \mathrm{ff}$. BSHG.

388 Insgesamt wurde mit einem Mehraufwand von 19,5 Mio. DM gerechnet; vgl. Begründung zum Regierungsentwurf, S. 66, BT, 3. Wp. 1957, Anlagen, Bd.67, Drs. 1799. 
Unfall Behinderten einen Rechtsanspruch einzuräumen. ${ }^{389}$ Doch die Bundesregierung erklärte selbst das lapidar für „zu weitgehend“ und verwies auf die Möglichkeiten der Kann-Bestimmungen. ${ }^{390}$ Auch gegenüber dem Fürsorgeausschuß des Bundestages lehnten die Beamten des Innenministeriums eine Ausweitung des Rechtsanspruchs ab, der auf Personen beschränkt werden müsse, „bei denen derzeit medizinisch im allgemeinen eine Hilfe überhaupt möglich sei“. ${ }^{391}$ Allerdings geriet das Ministerium dadurch in beträchtlichen Erklärungsnotstand, gehörten doch nach offizieller Begründung zum bevorrechtigten Behindertenkreis ausdrücklich nur „Personen mit einem geminderten Intelligenzgrad, nicht aber Geisteskranke oder Geistesschwache“.392

Eine solche Unterscheidung war nach Meinung vieler Mediziner und Sozialarbeiter angesichts neuer Rehabilitationsmethoden auch für geistig Behinderte nicht haltbar und würde in der Praxis zu erheblichen Schwierigkeiten führen. Vor allem ein im Frühling 1959 unter dem Vorsitz von Muthesius im DV zusammen mit weiteren Organisationen gegründeter kleiner „Aktionsausschuß zur Verbesserung der Hilfe für psychisch Kranke“, der auf dem Fürsorgetag im Oktober 1959 diesen Behinderten auch eine größere öffentliche Plattform verschafft hatte ${ }^{393}$, forderte wie übrigens auch der nordrhein-westfälische Sozialminister einen Rechtsanspruch für fast alle geistig/psychisch Behinderten und fand dabei die offizielle Unterstützung des DV. ${ }^{394}$ Auch der Ausschluß der nach inneren Organerkrankungen Körperbehinderten wurde von vielen Seiten als nicht nachvollziehbar kritisiert. ${ }^{395}$ Obwohl auch ein Verwaltungsplanspiel in Nordrhein-Westfalen die Abgrenzungsschwierigkeiten in der Praxis belegt hatte, trugen jedoch der Gesundheitsausschuß und der Fürsorgeausschuß des Bundestages die Regierungslinie mit, denn es könne „der Schritt in das Neuland der Eingliederungshilfe nur behutsam gegangen werden“.396 Im Bundestagsplenum schließlich wurde die Regelung ohne Aussprache beschlossen und auch vom Bundesrat nicht mehr aufgegriffen.

\footnotetext{
389 Vgl. Stellungnahme des Bundesrates zu $₫ 37$ des Regierungsentwurfs, S. 72, ebenda.

390 Auffassung der Bundesregierung zur Stellungnahme des Bundesrates, S. 84, ebenda.

391 Vgl. Sitzung des Ausschusses für Kommunalpolitik und öffentliche Fürsorge am 23.2. 1961, PA, Gesetzesmaterialien III/349 A 2.

392 Vgl. Begründung des BSHG-Regierungsentwurfs, S. 46, BT, 3. Wp. 1957, Anlagen, Bd. 67, Drs. 1799.

393 Vgl. NDV 40 (1960), S. 110; Fürsorge in der gewandelten Welt, S. 195ff., $234 \mathrm{f}$.

394 Vgl. die Berichte über die Sitzungen des Aktionsausschusses am 1.6. und 17.11.1960 sowie 16.11.1961, ADW, HGSt, SP-S XXIII d: 9-1/1; Änderungsvorschläge Prof. von Baeyer vom 7.10.1960, BAK, B 106/9786/2; Stellungnahme des DV zum Regierungsentwurf [o.D.] sowie die abschließenden Vorschläge des Aktionsausschusses dazu, PA, Gesetzesmaterialien III/349 A3, und die Vorschläge des nordrhein-westfälischen Sozialministers auf Grund des Verwaltungsplanspiels vom 2.2.1961, PA, Gesetzesmaterialien III/349 A4; NDV 41 (1961), S. 295.

395 Vgl. Bericht über die Sitzung des DV-Fachausschusses V am 2.6.1960, ADW, HGSt, SP-S XXIII c V/1; undatierte Notizen Collmers zum Regierungsentwurf, ADW, HGSt 3927.

396 So der CDU-Abgeordnete Maucher im Fürsorgeausschuß am 23.2.1961; vgl. Kurzprotokolle der Sitzungen des Ausschusses für Gesundheitswesen am 21.9.1960 sowie des Ausschusses für Kommunalpolitik und öffentliche Fürsorge am 10.11.1960 und 23. 2.1961, PA, Gesetzesmaterialien III/349 A1 bzw. A2.
} 
Doch der Bundestagsausschuß initiierte auch einige Verbesserungen zugunsten der Behinderten ${ }^{397}$ : Wie von vielen Seiten, darunter auch vom DV und der Bundesvereinigung der Kommunalen Spitzenverbände gefordert, wurde als Ziel der Eingliederungshilfe nun doch wieder auch die „Teilhabe am Leben in der Gemeinschaft" ausdrücklich im Gesetz genannt ${ }^{398}$. Außerdem wurde, wie erwähnt, für häuslich gepflegte Schwerbehinderte ein Pflegegeld von 100 DM monatlich eingeführt. ${ }^{399}$

Im Bundestag selbst standen wieder einmal die ärztlichen Belange im Mittelpunkt, zu deren Speerspitze sich abermals die FDP machte. In einer polemischen Stellungnahme wandte sich die Arbeitsgemeinschaft der fachärztlichen Berufsverbände Mitte Januar 1961 vor allem gegen die Kompetenzen der Gesundheitsämter, die unklare Einbeziehung der von einer Behinderung bedrohten Personen und die ärztliche Meldepflicht von ungenügend versorgten geschäftsunfähigen Körperbehinderten, Blinden etc. Das bedeute letztlich eine „gesundheitsbehördliche Erfassung eines uferlos ausgeweiteten Personenkreises“ und die „Aufhebung der Behandlungsfreiheit der Ärzte“. 400 Daraus sprachen ebenso die Furcht vor unkontrollierter amtsärztlicher Einmischung und einzelne groteske Erfahrungen mit dem KBG, das „,bereits zur Einbeziehung der leichten Fuß- und Haltungsschäden geführt" habe ${ }^{401}$, wie die aus standespolitischen Erwägungen überzeichnete Sorge, Patienten an künftig angeblich erforderliche öffentliche Ambulatorien zu verlieren. ${ }^{402}$ Nachdem aber der Fürsorgeausschuß weder den Kreis der von einer Behinderung Bedrohten näher definieren noch die Beratungskompetenzen von Landesarzt und Gesundheitsämtern einschränken wollte, lehnte auch die Bundestagsmehrheit entsprechende Anträge der FDP-Fraktion ab. ${ }^{403}$ Diese konnte allerdings zwei weitere wichtige Anliegen der Ärztevereinigung doch noch durchsetzen: Die freie Arztwahl auch bei der Eingliederungshilfe wurde festgelegt und, vor allem,

397 Vgl. zum Folgenden den Schriftlichen Bericht des Ausschusses für Kommunalpolitik und öffentliche Fürsorge vom 25.4.1961, S.6ff., BT, 3. Wp. 1957, Anlagen, Bd.74, Drs. 2673.

398 \39 Abs. 2 BSHG; vgl. ferner Stellungnahmen der Bundesvereinigung der Kommunalen Spitzenverbände vom 31.5.1960, des DV [o.D.], PA, Gesetzesmaterialien III/349 A3, sowie von Muthesius vor dem Bundestagsfürsorgeausschuß am 25.1.1961, ebenda, A 2.

399 Vgl. \$S 69, 80 BSHG.

400 Stellungnahme der Arbeitsgemeinschaft der fachärztlichen Berufsverbände vom 13.1. 1961, PA, Gesetzesmaterialien III/349 A 4 (Hervorhebung im Original).

401 Ebenda.

402 Die Stellung der Gesundheitsämter war im Laufe des Gesetzgebungsverfahrens ohnehin immer weiter geschwächt worden. So war die ursprünglich vorgesehene Zuständigkeit des Gesundheitsamts für die Aufstellung eines Rehabilitationsplans auf Antrag des Bundesrates fallengelassen worden; vgl. \$ 61 BSHG-Entwurf 7/1958, BAK, B 106/20643; Stellungnahme des Bundesrates zu $\ 43$ des Regierungsentwurfs 1960, BT, 3. Wp. 1957, Anlagen, Bd. 67, Drs. 1799; \46 BSHG.

${ }^{403}$ Vgl. den Schriftlichen Bericht des Ausschusses für Kommunalpolitik und öffentliche Fürsorge vom 25.4.1961, S.11f., BT, 3. Wp. 1957, Anlagen, Bd. 74, Drs. 2673; Bundestagssitzung am 4.5.1961, S. 9073ff., BT, 3. Wp. 1957, Sten. Ber., Bd. 49; Änderungsanträge der FDP-Fraktion vom 2. u. 3.5.1961, Umdrucke 888, 901, PA, Gesetzesmaterialien III/349 A 2. 
die ärztliche Meldepflicht praktisch aufgehoben. ${ }^{404}$ Ein ursprüngliches Kernanliegen der Reformer der Körperbehindertenfürsorge, die umfassende Meldepflicht im Interesse der Prävention, die schon im KBG nicht erreicht worden war, war mit dem BSHG vorerst aufgegeben worden.

Mit dieser Einschränkung war aufs Ganze gesehen doch das Ziel, das von vielen Zeitgenossen als „Modellgesetz“ begriffene Körperbehindertengesetz in das BSHG zu übernehmen und seine Neuerungen auf weitere Hilfekomplexe zu übertragen, gelungen. Den ursprünglich vorgesehenen Weg, die Hilfen nach dem KBG auf möglichst alle Behinderten auszudehnen, waren die Gesetzesmacher aus primär finanziellen Gründen allerdings nicht $\mathrm{zu}$ Ende gegangen und hatten lieber weiterhin definitorische Unklarheiten und sachlich kaum begründbare Ungleichbehandlungen in Kauf genommen. ${ }^{405}$ Erst 1974 bezog der Gesetzgeber neben erheblichen weiteren Verbesserungen alle Behinderten in den Rechtsanspruch ein und ließ ihnen die besondere Einkommensgrenze zugute kommen, so daß fast 16 Jahre nach Vorlage des ersten Referentenentwurfs dessen Konzeption doch noch verwirklicht wurde. ${ }^{406} \mathrm{Daß}$ aus Sicht der Träger der Sozialhilfe die ursprünglichen finanziellen Bedenken nicht unbegründet waren, belegt die Tatsache, daß die Eingliederungshilfe für Behinderte seit $1963 \mathrm{zu}$ den Hilfen mit besonders hohen Kostenzuwächsen gehörte. So beliefen sich die Kosten für diese Hilfe im Jahr 2000 auf rund 17,8 Mrd. DM für rund 525100 Hilfeempfänger. ${ }^{407}$ Damit bildete die Eingliederungshilfe nach Umfang und Bedeutung neben der Hilfe zur Pflege einen der beiden Schwerpunkte der Hilfe in besonderen Lebenslagen. ${ }^{408}$

\section{Exkurs: Hilfe für Blinde}

Für eine Behindertengruppe waren jedoch noch weitere besondere Maßnahmen der Sozialhilfe vorgesehen: für die Blinden. Diese hatten seit 1949 nämlich im sozialen Sicherungssystem der Bundesrepublik eine gewisse Sonderstellung erlangt, die einerseits der Schwere ihrer Behinderung und der vielfach notwendigen Fremdhilfe geschuldet war, sich andererseits aber wohl auch den im Vergleich zu anderen Sinnesgeschädigten besseren öffentlichen Artikulationsmöglichkeiten und einer effektiven Lobbyarbeit verdankte.

${ }^{404}$ Vgl. $\ 37$ Abs. 4 und $\ 124$ BSHG. Neben der ärztlichen Schweigepflicht und möglichen Belastungen für das Verhältnis zu ihren Patienten hatte die Ärztevereinigung auch die Gefahr von Regreßansprüchen geltend gemacht; vgl. Stellungnahme der Arbeitsgemeinschaft der fachärztlichen Berufsverbände vom 13.1.1961, S.11f., PA, Gesetzesmaterialien III/349 A 4.

405 So hörten die Streitigkeiten mit den Krankenkassen nach Inkrafttreten des BSHG keineswegs auf; ebenso schwierig blieb die schulische Versorgung von behinderten Kindern aus Mittelschichtsfamilien. Kritisch insgesamt der damalige westfälische Landesarzt Herbig, Problematik.

406 Vgl. das Dritte Änderungsgesetz vom 25.3.1974, BGBl. I S. 777.

407 Vgl. Statistisches Bundesamt, Fachserie 13, Reihe 2, 2000, S. $112 \mathrm{ff} ., 133$.

408 Vgl. Schellhorn, Bundessozialhilfegesetz, 2002, S. 10. 
Mitte der fünfziger Jahre gab es schätzungsweise 42-43500 Blinde in der Bundesrepublik, darunter ca. 7500 Kriegsblinde. ${ }^{409}$ Der Anteil der bereits blind Geborenen oder in der Kindheit Erblindeten war mit allenfalls einem Viertel vergleichsweise gering und ging dank medizinischer Fortschritte weiter zurück, während die Zahl der Späterblindeten (abgesehen von den Kriegsblinden) vor allem infolge der steigenden Lebenserwartung zunahm. ${ }^{410} 1956$ waren $65-75 \%$ der sogenannten Zivilblinden über 50 Jahre alt und Rentner der Sozialversicherung. Entsprechend gering war 1957 mit rund 10-11000 die Zahl der voll erwerbstätigen Blinden (einschließlich Kriegsblinden), von denen überdies noch etwa ein Drittel in traditionellen, immer weniger konkurrenzfähigen Blindenhandwerken (Bürstenmacher, Korb- oder Mattenflechter u.ä.) tätig war; hinzu kamen modernere, aber auch nicht sehr viel einträglichere Tätigkeiten als Industriearbeiter, Stenotypisten, Telefonisten, Masseure sowie eine kleine Gruppe von Verwaltungsbeamten, Lehrern etc. Organisatorische und recht einflußreiche Vertretung der Zivilblinden war der 1912 gegründete Deutsche Blindenverband (DBV), der 1956 rund fünf Sechstel (30000) aller Zivilblinden erfaßte.

Die Sozialleistungen für Blinde waren sehr ungleich verteilt: Während die infolge der neuartigen Gasangriffe bereits seit dem Ersten Weltkrieg beträchtliche Zahl der Kriegsblinden und die bei Arbeitsunfällen Erblindeten neben den Versorgungsrenten ein Pflegegeld zum Ausgleich ihrer Mehraufwendungen erhielten und auch im Rahmen des Lastenausgleichs solche Leistungen vorgesehen waren ${ }^{411}$, blieben die übrigen sogenannten Zivilblinden auf die öffentliche Fürsorge und deren Hilfsbedürftigkeits-Grenzen verwiesen, zumal auch blinde Sozialrentner keine zusätzlichen Leistungen erhielten. Bereits Ende der zwanziger Jahre waren Bestrebungen der Blindenorganisationen nach einer einkommensunabhängigen Blindenrente von der Reichsregierung aus Furcht vor ähnlichen Forderungen anderer Behindertengruppen und allgemeinen finanziellen Bedenken abgelehnt worden. ${ }^{412}$

Nach 1945/49 war die Interessenlage durchaus ähnlich, durch verfassungsmäßige Vorbehalte allerdings noch komplizierter: Anders als die Ländersozialminister im Oktober 1949 vertrat nämlich das Bundesinnenministerium die Rechtsmeinung, für eine versorgungsrechtliche Regelung habe der Bund keine Gesetzgebungskompetenz, diese liege vielmehr bei den Ländern. ${ }^{413}$ Tatsächlich führten viele von diesen im Laufe der fünfziger Jahre ein aus Landesmitteln zu finanzierendes Blindenpflegegeld ein, das aber hinsichtlich Höhe, Berücksichtigung an-

409 Vgl. Bierfelder/Sweede, Überblick, S. 279; Achinger, Rolle, S. 341; NDV 37 (1957), S. 49; Gottwald, Situation.

410 Nach einer Erhebung für Westfalen-Lippe im Jahr 1947 waren von 2630 Blinden 28\% Kriegsblinde, $11 \%$ Geburtsblinde und $16,2 \%$ bis zu ihrem fünfzehnten Lebensjahr erblindet; vgl. NDV 30 (1950), S. 106. 1960 schätzte eine wissenschaftliche Arbeit die Zahl der von Geburt oder Kindheit an Blinden in der Bundesrepublik auf 6500, der Gehörlosen hingegen auf 27000 ; vgl. Edelmann, Zahl, S. 92.

411 Vgl. \\ 267-269 LAG, \35 BVG sowie $\ 558 \mathrm{c}$ RVO.

412 Vgl. NDV 30 (1950), S. 105.

413 Vgl. Staatssekretär Bleek vom BMI in der Sitzung des Bundestages am 10.10.1951, BT, 1. Wp. 1949, Sten. Ber., Bd. 9, S. 6769. 
derweitigen Einkommens und in seinem Verhältnis zu den Leistungspflichten der öffentlichen Fürsorge sehr unterschiedlich ausfiel. ${ }^{414}$

Bestrebungen der Zivilblinden für eine bundesweite Gleichstellung mit den Kriegsblinden stießen zwar durchaus auf Sympathie bei sämtlichen Bundestagsfraktionen, und 1953 wurden immerhin die Blinden als einzige Gruppe der nicht durch Krieg, Arbeitsunfall oder NS-Verfolgung Behinderten in das Schwerbeschädigtengesetz einbezogen. ${ }^{415}$ Die SPD unterstützte die Forderung des DBV nach einer versorgungsrechtlichen Lösung durch ein Bundesblindengesetz, da schließlich auch die nicht oder nur teilweise fürsorgebedürftigen, unter erheblichen Kraftanstrengungen erwerbstätigen oder nur kleine Sozialversicherungsrenten erhaltenden Blinden weiter Unterstützung benötigten. ${ }^{416}$ Zwar hoffte auch der DBV-Vorsitzende Alfons Gottwald, daß etwa die noch in den Kinderschuhen steckende elektronische Datenverarbeitung den Blinden neue Berufsfelder wie den „Programmeur an einer Elektronenrechenmaschine“ 417 erschließen könne, doch insgesamt seien für Blinde nur wenige Berufe überhaupt zugänglich und der gravierende Einkommensrückstand gegenüber den Sehenden niemals aufzuholen, so daß alle Blinden einen Anspruch auf ein Blindengeld zum Ausgleich ihrer Mehrbelastungen sowie auf Aus- und Fortbildung, auf Rehabilitation und Erholungsmaßnahmen haben müßten. ${ }^{418}$

Demgegenüber hielt die Regierungskoalition angesichts der Verfassungsvorgaben nur eine fürsorgerechtliche Lösung für möglich. ${ }^{419}$ Dieser Weg wurde 1953 dann auch mit dem FÄG beschritten, das die Blinden gegenüber anderen körperlich Behinderten privilegierte und erstmals einem größeren Kreis von Zivilblinden eine Pflegezulage ohne Kostenersatzpflicht einräumte. Zusätzlich wurde erwerbstätigen Blinden noch ein Mehrbedarf von mindestens 40\% ihres Erwerbseinkommens bzw. mindestens 40 DM zuerkannt, so daß (bei einem durchschnittlichen Richtsatz von 50 DM) etwa auch einem Blinden mit einem bescheidenen Monatseinkommen von 260 DM eine gewisse Pflegezulage zustand. ${ }^{420}$ Auf dem Weg zu einem Pflegegeld für Zivilblinde war damit zwar ein entscheidender Schritt getan,

414 Zum Stand der verschiedenen Länderregelungen vgl. Stellungnahme des Blindenverbands zum BSHG-Regierungsentwurf o.D. [27.10.1960], PA, Gesetzesmaterialien III/349 A3, Entschließung des DBV vom 31.1.1961, ebenda, B; ferner die Niederschrift über die Besprechung der Länderfürsorgereferenten am 12./13.6.1958, BAK, B 106/20132. Zur Rechtslage in Bayern siehe Jehle, Fürsorgerecht, S. 190, 816f.; zum Saarland NDV 37 (1957), S. 33.

415 Vgl. \1 Abs. 2 des Schwerbeschädigtengesetzes vom 16.6.1953, BGBl. I S. 389.

416 Vgl. die SPD-Abgeordnete Clara Döhring am 12.6.1953 vor dem Bundestag, BT, 1. Wp. 1949, Sten. Ber., Bd. 16, S. 13408f.; entsprechende frühere Initiativen der SPD-Fraktion: Anfrage vom 12.5.1950, Interpellation vom 8.7.1951, BT, 1. Wp. 1949, Anlagen, Bd.4, Drs. 950; sowie Bd.12, Drs. 2435.

417 Gottwald, Situation, S. 117.

418 Nach einer Erhebung in Bayern von 1952 verdienten blinde Sozialversicherte mit monatlich rund 140 DM im Durchschnitt nur die Hälfte der sehenden Versicherten; vgl. Gottwald, Situation, S. 117.

419 Vgl. etwa die Berichterstatterin Niggemeyer am 12.6.1953 vor dem Bundestag, BT, 1. Wp. 1949, Sten. Ber., Bd. 16, S. 13404.

420 Vgl. Döhring, Jahre, S. 209. 
doch das Ziel einer festen, einkommensunabhängigen Hilfe war noch lange nicht erreicht: 1958 erhielten etwa in Schleswig-Holstein gut 70\% von 1000 Zivilblinden ein Pflegegeld der öffentlichen Fürsorge, aber aufgrund der Anrechnungsbestimmungen nur jeder fünfte in voller Höhe. ${ }^{421}$

Bei den Vorarbeiten für das neue Bundesfürsorgegesetz plante die Sozialabteilung zunächst, diese Regelungen beizubehalten. ${ }^{422}$ Doch bereits der Teilentwurf vom Mai 1956 führte neben besonderen Bestimmungen für Körperbehinderte, Tuberkulöse und Kriegsopfer auch solche für Blinde an, die bereits eine besondere Einkommensgrenze vorsahen. ${ }^{423}$ Dies war vermutlich bereits ein Reflex auf Forderungen der SPD, bei der Rentenreform im Rahmen der Sozialversicherung ein Pflegegeld für pflegebedürftige, also auch blinde Rentner einzuführen. Diese Forderung wurde von der Regierungskoalition abgelehnt; statt dessen beauftragte der Bundestag im Januar 1957 die Bundesregierung einstimmig, mit den Ländern nach einer Vereinheitlichung der Blindengeld-Regelungen zu suchen. ${ }^{424}$ Aus Sicht des Bundesinnenministeriums konnte dies nur im Rahmen des künftigen Fürsorgerechts geschehen, doch war man bereit, mit Rücksicht auf „den politischen Charakter dieser Frage" bis an die Grenzen des gegenüber den skeptischen kommunalen Trägern Vertretbaren zu gehen ${ }^{425}$ : Auf dem Fürsorgetag im November 1957 kündigte Scheffler eine generelle besondere „Blindenhilfe“ zum Ausgleich aller durch die Blindheit bedingten Mehraufwendungen mit einer Einkommensgrenze an, die über der für die meisten Hilfen in besonderen Lebenslagen geltenden liegen sollte. ${ }^{426}$

Diese auch vom Beirats-Fürsorgeausschuß gestützten Pläne stießen jedoch auf deutlichen Widerstand Gottwalds, dem an einem umfassenden Fürsorgegesetz die Nähe der Blindenhilfe zu Hilfen für andere Personengruppen, „insbesondere der Gefährdeten und der Nichtseßhaften“ deutlich mißfiel.427 Die Blindenfürsorge, bislang „immer noch ein Anhängsel der "Armenfürsorge" moderner Prägung" müsse endlich wie etwa die Körperbehindertenfürsorge aus diesem Kontext befreit und zumindest ein eigenes Fürsorgegesetz für Blinde geschaffen werden. ${ }^{428}$

${ }^{421}$ Vgl. Niederschrift über die Besprechung der Länderfürsorgereferenten am 12./13.6. 1958, BAK, B 106/20132.

422 Vgl. [Konzept] „C. Sozialhilfe“ vom 29.11.1955, BAK, B 106/20648; Anlage „Sozialunterstützung“ vom 10.1.1956 für Abteilungsleiter; Anlage zu Besprechung mit Abteilungsleiter am 7.2.1956, BAK, B 106/9688.

423 Vgl. SS 82-85 des Teilentwurfs vom 25. 5.1956, BAK, B 106/20648.

424 Vgl. die Sitzungen des Bundestages am 16., 18. und 21.1.1957 sowie Umdruck 912, BT, 2. Wp. 1953, Sten. Ber., Bd. 34, S. 10182, 10413f., 10575ff., 10599, 10617.

425 Gottschick im Arbeitsausschuß für Fragen der Fürsorge am 21./22. 2.1958, Niederschrift, ADW, HGSt 6769.

426 Vgl. Gerhard Scheffler, Neuordnung, S.36f.; entsprechend der BSHG-Entwurf vom 29.11.1957, BAK, B 106/20643; ferner Vermerk Referat V A 4 vom 7.5.1957, BAK, B 106/9789/2.

427 Vermerk Referat V A 4 vom 27.5.1958, BAK, B 106/9686; vgl. Niederschrift über die Sitzung des Arbeitsausschusses für Fragen der Fürsorge am 21./22. 2. 1958, ADW, HGSt 6769.

428 Gottwald, Situation, S.117; vgl. auch Neuordnung des Fürsorgerechts, S.414f.; Die Blindenwelt 1958, H. 1, S. 3f.; H. 4, S.1f. 
Tatsächlich hatte der rührige DBV-Vorsitzende den Entwurf für ein Bundesblindengesetz dem Bundestag zugeleitet, referierte darüber vor dem Sozialpolitischen Arbeitskreis der CDU/CSU und versuchte in hartnäckigen Verhandlungen mit dem Bundesinnenministerium im Herbst 1958, doch noch den Standpunkt des DBV durchzusetzen. ${ }^{429}$ Doch die Sozialabteilung lehnte aus den bekannten Gründen ein solches Sondergesetz ab und legte statt dessen mit dem BSHG-Referentenentwurf vom Juli 1958 Regelungen für eine erweiterte „Blindenhilfe“ vor.

Danach hatten Blinde (außerhalb von Anstaltspflege) ab dem 18. Lebensjahr Anspruch auf monatlich 150 DM, 6-17jährige Blinde erhielten die Hälfte. Kleinere Kinder fielen unter die allgemeine „Hilfe zur Pflege“. .30 Wie im bisherigen Fürsorgerecht galt als blind auch, „wer eine so geringe Sehkraft hat, daß er sich in einer ihm nicht vertrauten Umgebung ohne fremde Hilfe nicht zurechtfinden kann “. ${ }^{431}$ Innovativ und für die Fürsorge untypisch war dabei nicht die Höhe dieser Einkommenshilfe selbst, die dem aktuellen Stand des Pflegegeldes nach dem Bundesversorgungsgesetz entsprach: Schon das bisherige Pflegegeld der Fürsorge in Höhe des doppelten Richtsatzes lag im Bundesdurchschnitt bei 140 DM.432 Entscheidend war vielmehr die nun auch vom Richtsatz gelöste Standardisierung durch einen festen Geldbetrag ohne Rücksicht auf die individuellen wirtschaftlichen Verhältnisse. Der Beirats-Fürsorgeausschuß und auch der DV-Fachausschuß I hätten allerdings lieber die automatische Koppelung an das jeweilige Pflegegeld für Kriegsblinde gesehen, aber so weit wollte das Innenministerium die Annäherung an die Versorgung dann doch nicht treiben. ${ }^{433}$ Daneben war die hohe Einkommensgrenze die entscheidende geplante Neuerung: Auch für die Blindenhilfe sollte diese nämlich bei $600 \mathrm{DM}$ brutto plus Familienzuschlägen liegen, so daß auch die meisten Sozialrentner dieses Geld erhalten würden. ${ }^{434}$ Neben diesen Sonderregelungen konnten Blinde natürlich auch die Eingliederungshilfen, die für sämtliche Behinderten vorgesehen waren, erhalten. Jetzt signalisierte der DBV, daß man die Forderung nach einem Sondergesetz fallen lassen könne, wenn nur die Einkommensgrenze gänzlich entfiele. ${ }^{435}$ Doch hier lenkte die Sozialabteilung nicht ein und erklärte, es widerspreche „fürsorgerechtlichen Grundsätzen [...], bei der Gewährung einer Einkommenshilfe eigenes Einkommen völlig unberücksichtigt zu lassen". ${ }^{436}$

Nichtsdestoweniger liefen die kommunalen Spitzenverbände - und zwar einhellig - gegen die geplante Regelung Sturm: Ihre recht großzügig veranschlagten

${ }^{429}$ Vgl. Vermerke Referat V A 4 vom 11.6. und 9.9.1958, BAK, B 106/9686; Die Blindenwelt 2 (1960), S. 1.

430 Vgl. \ 66 des BSHG-Entwurfs 7/1958, BAK, B 106/20643.

431 \28 Abs. 3 ebenda.

432 Vgl. Gottschick, Referentenentwurf, S. 19.

433 Vgl. NDV 38 (1958), S.310; Bericht über die Sitzung des Fachausschusses I am 24.10. 1958, ADW, HGSt, SP-S XXIIIc I/1.

434 Vgl. $\ 80$ des BSHG-Entwurfs 7/1958, BAK, B 106/20643; 1958 betrug in der Arbeiterrentenversicherung die Durchschnittsrente für Versicherte 144 DM, für Witwen 100 DM; vgl. Ritter/Niehuss, Wahlen, S.70, Tabelle 1.38.

435 Vgl. Stellungnahme des DBV vom 26. 9.1958, BAK, B 106/20644.

436 Begründung zum BSHG-Entwurf 3/1959, Teil B, S.36, BAK, B 106/20646. 
Schätzungen der künftigen Mehrbelastungen reichten von 57,6 bis 70 Mio. DM jährlich, was eine Verdreifachung der bisherigen Aufwendungen für Blinde bedeutet hätte. ${ }^{437}$ Angesichts ihres „Versorgungscharakters“ müßte diese Hilfe von Bund oder Ländern finanziert und daher aus dem BSHG gestrichen werden.438 Trotz ähnlicher Bedenken bei vielen Ländervertretern und im DV trugen diese dagegen den Entwurf mit, lehnten aber ebenfalls den Wegfall jeglicher Einkommensgrenzen strikt ab. ${ }^{439}$ Allerdings schätzten die Länder nach Abzug ihrer eigenen Pflegegeldleistungen den jährlichen Mehraufwand für die Sozialhilfeträger auch nur auf 25 Mio. DM, womit die Blindenhilfe allerdings immer noch eine der teuersten Hilfen überhaupt sein würde. 440

So blieb die Blindenhilfe im Regierungsentwurf vom Februar 1960 im wesentlichen unverändert bestehen. ${ }^{441}$ Anders als bei den meisten anderen Hilfen hatten sich aufgrund der politischen Stimmungslage im Bundestag in diesem Fall die Kommunen mit Forderungen nach Leistungsreduktionen also nicht durchsetzen können. ${ }^{442}$ Im Gegenteil: Trotz ihrer Intervention beim zuständigen Unterausschuß des Bundesrates Anfang März ${ }^{443}$ mußten die kommunalen Spitzenverbände sogar hinnehmen, daß der Bundesrat am 18. März 1960 für die Blindenhilfe eine eigene Einkommensgrenze von 1000 DM (plus Familienzuschlägen) festlegte, denn nur so werde der mit der Blindenhilfe „verfolgte Zweck befriedigend [...] sichergestellt werden können“. 444 Auf diese Weise würden nach Schätzung Gottwalds weitere 1000 Zivilblinde in den Genuß der Hilfe kommen. ${ }^{445}$ Die Bundes-

437 1958: 32,7 Mio. DM, vgl. Begründung zum Regierungsentwurf vom Februar 1960, S. 66, BT, 3. Wp. 1957, Anlagen, Bd.67, Drs. 1799. Der DST-Sozialausschuß bezog in seiner vorläufigen Stellungnahme zur Neuordnung des Fürsorgerechts (Teil II) vom 20.8. 1958, LAB, B Rep. 142-9, 1283, praktisch alle rund 40000 Blinden mit ein, die bayerischen kommunalen Spitzenverbände gingen davon aus, daß keiner der ca. 32000 Zivilblinden die Einkommensgrenze überschreiten würde; vgl. Stellungnahme vom 14.10. 1958, BAK, B 106/9686.

438 Vgl. Stellungnahmen des DLT vom 17.11.1958, des DST vom 27.11.1958, des DGT vom 1.12.1958, BAK, B 106/9686.

439 Vgl. Niederschrift über die Besprechung mit Vertretern der obersten Landessozialbehörden am 21./22. 10.1958, LAS Abt. 761 Nr. 8874; Äußerungen aus den DV-Fachgremien [5.11.1958] zu $\ 80, \mathrm{BAK}, \mathrm{B} 106-9686$, Stellungnahme der AG der LFV vom 17.12.1958, BAK, B 106/20644.

440 Nach Stand des Regierungsentwurfs; vgl. die Begründung zum Regierungsentwurf vom Februar 1960, S. 66, BT, 3. Wp. 1957, Anlagen, Bd.67, Drs. 1799.

${ }^{441} \mathrm{Vgl}$. die $\$ \mathbb{S} 22,64,77,92$ des Regierungsentwurfs, ebenda.

442 Vgl. Vermerk Oel vom 28. 6.[1959] über den BSHG-Entwurf 3/1959, LAB, B Rep. 1429, 1284; Wormit an BMI, 18.6.1959, BAK, B 106/20644; Niederschrift über die Sitzung des DLT-Sozialausschusses am 5./6.11.1959, BAK, B 172/444-02/2.

${ }^{443}$ Vgl. die namens aller kommunalen Spitzenverbände abgegebene vorläufige DST-Stellungnahme vom 2.3.1960, LAB, B Rep. 142-9, 1284.

${ }^{444}$ Stellungnahme des Bundesrates zum Regierungsentwurf zu $\ 77$, S. 75, BT, 3. Wp. 1957, Anlagen, Bd.67, Drs. 1799; vgl. ferner Stellungnahme des Bundesratsausschusses für Innere Angelegenheiten vom 11.3.1960, S.27, BR 1960, Drucksachen, Drs. 53/1/60; Bundesratssitzung am 18.3.1960, S.340, BR 1960, Sten. Ber.

445 Vgl. Kurzprotokoll der Sitzung des Bundestagsausschusses für Kommunalpolitik und öffentliche Fürsorge am 27.10.1960, PA, Gesetzesmaterialien III/349 A1. 
regierung stimmte dem in der Sache ohne weiteren Kommentar zu; vermutlich war man im Bundesinnenministerium froh, den Forderungen der Blinden noch weiter entgegenkommen und gegenüber den Kommunen die Länder dafür verantwortlich machen zu können. ${ }^{446}$

Auch in der parlamentarischen Verhandlungsphase blieb die Blindenhilfe ein zentraler Diskussionspunkt. Dabei ging es weniger um eine weitere Änderung der Leistungsinhalte als um Prinzipienfragen, denn die Zahl der Zivilblinden, die mehr als 1000 DM verdienten, war nach allgemeiner Auffassung denkbar gering ${ }^{447}$, und das Bundesinnenministerium rechnete mit jährlichen Mehrkosten von ganzen 2,5 Mio. DM.448 Die Blindenorganisationen aber wollten endlich die Gleichstellung mit den Kriegsblinden, deshalb mußte die Einkommensgrenze fallen; daß deren Kostenersparnis mit den dauernden Einkommenskontrollen und dem Verwaltungsaufwand in keinem Verhältnis stünde, war nur ein zusätzliches Argument. ${ }^{449}$ Für die Kommunen war umgekehrt die als viel zu hoch kritisierte Einkommensgrenze der letzte Schutzwall vor dem gefürchteten „Einbruch“ von Versorgungsansprüchen in das Fürsorgerecht, zumal der Ausschluß anderer Schwerbehinderter von diesen Leistungen kaum lange aufrechtzuerhalten wäre. ${ }^{450}$ An dieser Auffassung änderte weder ein von dem bekannten Verwaltungsrechtler Ernst Forsthoff bereits 1954 verfaßtes Gutachten etwas, wonach bei Blindheit immer Hilfsbedürftigkeit gegeben, also auch eine einkommensunabhängige Blindenhilfe eine Fürsorgeleistung im Sinne des Grundgesetzes und damit der Gesetzgebungskompetenz des Bundes unterstellt sei. ${ }^{451}$ Noch die Tatsache, daß in der DDR seit 1959 ein abgestuftes Blindengeld (maximal: $120 \mathrm{M}$ ) ohne Einkommensgrenze eingeführt worden war. ${ }^{452}$

446 Vgl. Auffassung der Bundesregierung zur Stellungnahme des Bundesrates, S. 85, BT, 3. Wp. 1957, Anlagen, Bd.67, Drs. 1799; auch Gottschick, Entwurf, S. 143.

447 Laut Berechnung des Bayerischen Blindenbunds nur 100-110 von rund 30000 Zivilblinden; vgl. Anlage 4 zum Bericht über die Sitzung des DV-Fachausschusses I am 17./18.5. 1960, ADW, HGSt, SP-S XXV 1: 110-1/2.

448 Vgl. die Zusammenstellung des BMI, 2.1.1961, PA, Gesetzesmaterialien III/349 A3.

449 Vgl. Stellungnahme Gottwalds vor dem Bundestagsausschuß am 27.10.1960, ebenda; Entschließung des DBV vom 31.1.1961, Stellungnahme des Vereins blinder Geistesarbeiter Deutschlands vom Januar 1961 sowie Schreiben des DBV an die Mitglieder des Bundestagsausschusses vom 10.2.1961, ebenda, B.

450 Vgl. Keese vor dem Richtsatz-Arbeitskreis des DV am 16.5.1960, NDV 40 (1960), S. 279; Anlage 4 zum Bericht über die Sitzung des DV-Fachausschusses I am 17./18.5. 1960, ADW, HGSt, SP-S XXV 1: 110-1/2; Vermerk Oel dazu o.D. sowie Keese an Oel am 19.5.1960, LAB, B Rep. 142-9, 1256; Hoppe, Entwurf, S.359; Stellungnahme der Bundesvereinigung der Kommunalen Spitzenverbände vom 30.5.1960 zum Regierungsentwurf sowie Referate Oel (DST) und Possehl (DSB) vor dem kommunalpolitischen Bundestagsausschuß am 23.6.1960, PA, Gesetzesmaterialien III/349 A3. Entsprechende Wünsche anderer Gruppen in den Stellungnahmen des VdK vom 14.10.1960 und des Reichsbunds o.D., ebenda, sowie der Gesellschaft zur Förderung der Hör- und Sprachgeschädigten vom 24.11.1958, BAK, B 106/9686.

451 Ein Exemplar des Gutachtens in: AdsD, SPD-BTF 3. Wp., 134. Vgl. ferner Die Blindenwelt 1958, H. 4, S.1.

452 Vgl. Verordnung über die weitere soziale Sicherung der Blinden und anderer Schwerbeschädigter vom 18.6.1959, Gesetzblatt der DDR I S.606. 
Auch der Bundestagsausschuß für Kommunalpolitik und öffentliche Fürsorge hielt schließlich am Prinzip der Einkommensgrenze fest, erhöhte aber das Pflegegeld für erwachsene Blinde auf 200 DM, da mittlerweile schon der doppelte Regelsatz vielfach über $150 \mathrm{DM}$ und die Pflegezulage für Kriegsblinde bei $200 \mathrm{DM}$ lag. ${ }^{453}$ Die von der SPD geforderte Herabsetzung des Mindestalters auf zwei Jahre hingegen wurde unter Hinweis auf die Finanzlage vieler Gemeinden vom Ausschuß denkbar knapp sowie später auch vom Bundestag abgelehnt. ${ }^{454}$ So wurde die Blindenhilfe in der Fassung des Ausschusses Gesetz. ${ }^{455}$ Daß dieser Kompromiß allerdings praktischen Erfordernissen nicht gerecht wurde, belegt die Tatsache, daß sie bereits 1965 geändert wurde: Das Mindestalter der Blindenhilfe wurde auf 3 Jahre herabgesetzt, das Blindengeld auf 240 DM erhöht und auf Blinde in Anstalten und Heimen ausgedehnt. ${ }^{456}$ Später ist das Blindengeld mit Bezug auf die aktuellen Renten der Sozialversicherung dynamisiert worden und die untere Altersgrenze ganz entfallen; eine Einkommensgrenze allerdings gibt es nach wie vor. ${ }^{457}$ Insgesamt betrachtet belegt die Entwicklung der Blindenhilfe, daß auch auf dem Feld der dafür sonst wenig prädestinierten Fürsorge/Sozialhilfe "aktive Betroffene“ die sozialpolitische Problembearbeitung mittels effizienter Einflußnahme auf Parlament und Öffentlichkeit in ihrem Sinne beeinflussen können. ${ }^{458}$ Indiz dafür ist auch die Tatsache, daß alle, auch die neuen Bundesländer ein gesetzliches Landesblindengeld eingeführt oder andere bundesrechtliche Vorschriften vorrangig gegriffen haben. Dadurch hatte die einst so umstrittene Blindenhilfe des BSHG trotz weiter gestiegener Zahl von Blinden lange Zeit kaum mehr praktische Bedeutung. ${ }^{459}$ Erst infolge der Kürzung oder Streichung von Leistungen der Landesblindengelder in allerjüngster Zeit gewinnt die (mittlerweile in $\$ 72$ SGB XII geregelte) Blindenhilfe der Sozialhilfe wieder an Bedeutung. 460

${ }^{453}$ Für 6-17jährige die Hälfte. Außerdem schlug er eine günstigere Regelung des Mehrbedarfs bei der HLU vor; vgl. den Schriftlichen Bericht des Ausschusses vom 25.4.1960, S. 5, 7f., 21, 36f., 43, BT, 3. Wp. 1957, Anlagen, Bd. 74, Drs. 2673.

$454 \mathrm{Vgl}$. Kurzprotokoll der Sitzung des Ausschusses für Kommunalpolitik und öffentliche Fürsorge am 1.3.1961 sowie Änderungsantrag des SPD-Fraktion vom 2.5.1961, Umdruck 872, PA, Gesetzesmaterialien III/349 A2; Bundestagssitzung am 4.5.1961, S. $9075 f$., BT, 2. Wp. 1957, Sten. Ber., Bd. 49.

455 Vgl. $\int S$ 24, 67, 81, 100 BSHG.

456 Vgl. Gesetz zur Änderung und Ergänzung des BSHG vom 31. 8. 1965, BGBl. I S. 1027.

457 Zur Weiterentwicklung der Blindenhilfe seit 1961 vgl. Schellhorn, Bundessozialhilfegesetz, 2002, zu \$67 BSHG, S. 433.

458 Vgl. allgemein Schetsche, Karriere, S. 41.

${ }^{459} \mathrm{Im}$ Laufe des Jahres 2000 erhielten rund 6200 Personen Blindenhilfe in Höhe von 21,4 Mio. DM; vgl. Statistisches Bundesamt, Fachserie 13, Reihe 2, 2000, S. 90, 112.

460 Vgl. www.seh-netz.info/hilfen_soziales/soziales/blindenhilfe/4.htm. (21.09.2005) 